\title{
Adaptación de una corona metal cerámica como pilar de una prótesis parcial removible preexistente
}

Adaptation of a metal ceramic crown as an abutment for a pre-existing removable partial denture.

Melissa Torres Herbozo ${ }^{1, a, b}$, Martin Quintana del Solar ${ }^{1, a, c, d}$, Diana Castillo Andamayo ${ }^{2, a, d}$

\section{RESUMEN}

El presente artículo muestra el reporte de un caso clínico donde se confeccionó una corona metal cerámica que fue adaptada a una prótesis parcial removible pre existente. Por lo general el tratamiento para estos casos es la confección de una nueva prótesis parcial removible adaptada a la nueva corona, lo cual implica mayor tiempo de trabajo y costo para el paciente. Diversos materiales y técnicas pueden ser utilizados para la realización de la corona como por ejemplo coronas de aleaciones metálicas completas, coronas metálicas con revestimiento cerámico y coronas libres de metal incluso con técnica CAD/CAM. A continuación se detalla paso a paso la elaboración de una corona metal cerámica con su respectiva preparación biostática adaptada de acuerdo a la forma preexistente de la base metálica de la PPR utilizada por el paciente.

PALABRAS CLAVE: Cerámica, Dentadura Parcial Removible, Coronas dentales.

\section{SUMMARY}

The paper presents the report of a clinical case where a metal ceramic crown was adapted to a pre existing removable partial denture. Usually treatment for these cases is the preparation of a new removable partial denture adapted to the new crown, which means more clinical time and cost raise of the final procedure for the patient. Several materials and techniques could be used to fabricate the crown, for example complete crowns with metal alloys, metal ceramic crowns and metal free crowns even with CAD/CAM technique. We detail a step by step technique to fabricate a metal ceramic crown with its own biostatic preparations according to the pre existing

Programa de pos grado en Rehabilitación Oral de la Facultad de Estomatología, Universidad Peruana Cayetano Heredia. Lima, Perú. Departamento Académico de Odontología Social (DAOS) de la Facultad de Estomatología Roberto Beltrán, Universidad Peruana Cayetano Heredia. Lima, Perú.

Cirujano Dentista

Residente

Magister en Estomatología

Especialista en Rehabilitación Oral

Maestría en Estomatología 
metal framework of the patient's removable partial denture.

\section{KEY WORDS: Ceramics, removable partial denture, dental crowns.}

\section{INTRODUCCIÓN}

La restauración de un pilar de una prótesis parcial removible preexistente puede ser un procedimiento complejo y que requiere mucho tiempo. Muchos factores pueden hacer que los pilares que soportan a una PPR se deterioren, como por ejemplo caries, fracturas, síndrome de diente agrietado, trauma, o caries recidivante debajo de alguna corona o restauración defectuosa con un subsecuente tratamiento de conducto (1); en la literatura se describen numerosas técnicas de restauración para estos casos $(1,2)$, algunas describen procedimientos para restaurar coronas puramente metálicas (2), y otras metal-cerámicas, las cuales son más estéticas(1).

El propósito de este artículo fue describir una técnica para elaborar una restauración coronaria completa adaptada a una base metálica de prótesis parcial removible pre existente usando una corona metal cerámica respetando el descanso oclusal, la placa de contacto proximal, conector menor y ecuador protésico correspondiente.

\section{Caso clínico}

Paciente de sexo femenino de 62 años de edad en

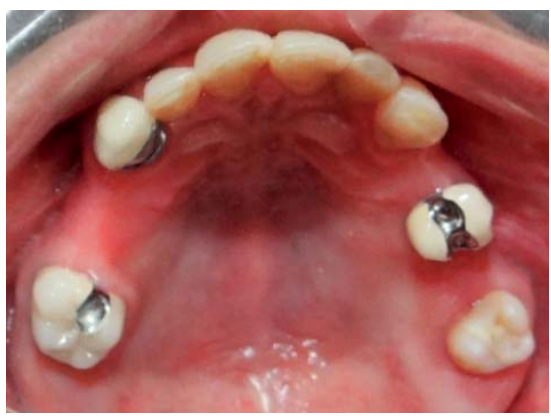

Figura 1. Maxilar Superior aparente buen estado general (ABEG) acude al Servicio del Postgrado de Rehabilitación Oral de la Facultad de Estomatología Roberto Beltrán de la Universidad Peruana Cayetano Heredia. Su motivo de consulta fue "Quiero ponerme una corona porque me han realizado una endodoncia”. La paciente da su consentimiento para realizar esta publicación académica. Al examen clínico intraoral presentaba coronas metal cerámica en pzas 15, 23, 26, reborde edéntulo superior a nivel de pzas 16, 14, 24, 25 (Figura 1) y en el maxilar inferior presentaba extremo libre unilateral lado izquierdo, así como restauraciones con amalgama en pza 46 y ionómero resina en pza 35 (Figura 2). Al examen radiográfico se observó tratamiento de conducto en buen estado en pza 35, siendo la rehabilitación de dicha pieza el motivo de consulta de la paciente. La paciente era portadora de una prótesis parcial removible dento-muco soportada inferior, la cual fue fabricada un año antes de la consulta (Figura 3). La PPR se presentó clínicamente aceptable y la paciente se encontró conforme con ella. La paciente no portaba PPR superior por haberla perdido.

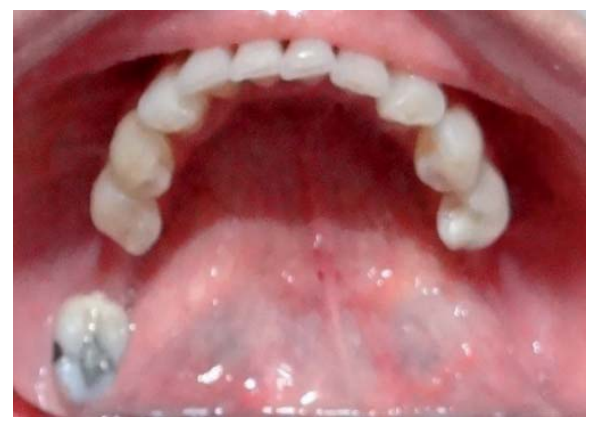

Figura 2. Maxilar Inferior

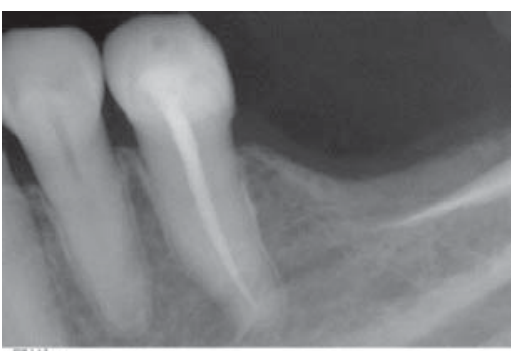

Figura 3. Radiografía Periapical pieza 35 
El diagnóstico de la paciente fue:

a) Gingivitis asociada a placa dental sin otro factor contribuyente

b) Deformidades mucogingivales en rebordes edéntulos: Seibert clase III en maxilar superior e inferior

c) Edentulismo parcial superior e inferior clase III subdivisión 2 y clase II subdivisión 1 respectivamente.

El tratamiento planteado fue la colocación de espigo de fibra de vidrio y corona metal cerámica en pza 35 la cual fue adaptada a la prótesis parcial removible pre existente. $\mathrm{Y}$ en el maxilar superior se propuso la confección de una prótesis parcial removible con base metálica dentosoportada.

A continuación se describe la técnica que se utilizó en la paciente para la confección de una corona metal cerámica adaptada a una prótesis parcial removible:

\section{Técnica}

Se evaluó la PPR existente y el diente pilar para asegurar que ambos son funcionales y con pronóstico favorable.

Una vez colocado el espigo pre fabricado de fibra de vidrio, se preparó el diente pilar con una terminación en bisel (145 grados) y se realizó el desgaste correspondiente a las paredes axiales y oclusales con los espacios adecuados para la correcta aplicación de los materiales (metal y porcelana) (Figura 4).

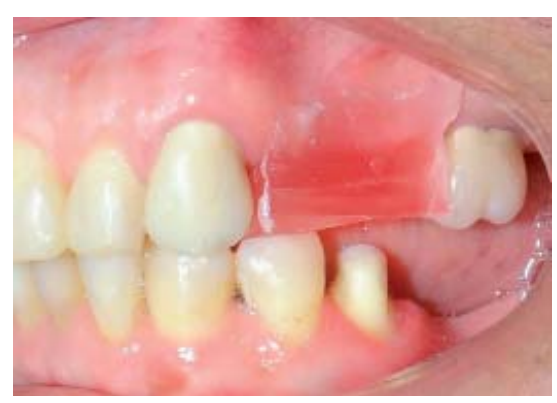

Figura 4. Preparación Dentaria

Se colocó la PPR con base metálica intraoralmente para asegurar el adecuado espacio para la futura corona metalceramica y la estabilidad de la base (Figura. 4).

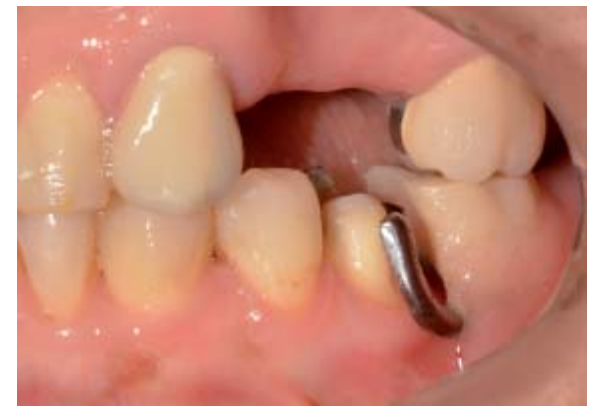

Figura 5. Verificación de espacio disponible

Se confeccionó la corona provisional utilizando acrílico Duralay color 62 realizando los rebasados necesarios utilizando la PPR aislada con vaselina por el interior hasta copiar fielmente el descanso oclusal, placa de contacto proximal, conector menor y zona retentiva del gancho de la prótesis parcial removible, se verificó el correcto asentamiento de la PPR. Se realizó el pulido del provisional con cauchos para acrílico y pasta pulidora, se cementó temporalmente el provisional (Figura 6).

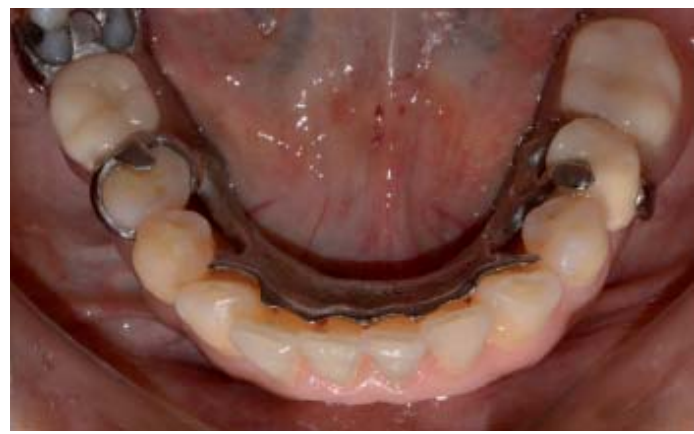

Figura 6. Corona provisional adaptada a prótesis parcial removible

Se tomó la impresión definitiva del pilar con silicona por condensación mediana (Thixoflex M, Zhermack). Se utilizó la técnica de copas de ripol con el respectivo arrastre de la copa con hidrocoloide irreversible con las proporciones que indica el fabricante (Tropicalgin, Zhermarck). Se realizó el vaceado de la impresión con yeso tipo IV (Fujirock, GC), se troqueló y delimitó.

Se Indicó al laboratorio dental que confeccione una cofia de acrílico de combustión completa (Duralay) sobre el muñón de yeso, sin añadir las compensaciones comúnmente realizadas. Esta cofia de acrílico Duralay se probó en boca del paciente, se verificó sellado marginal (Figura 7). 


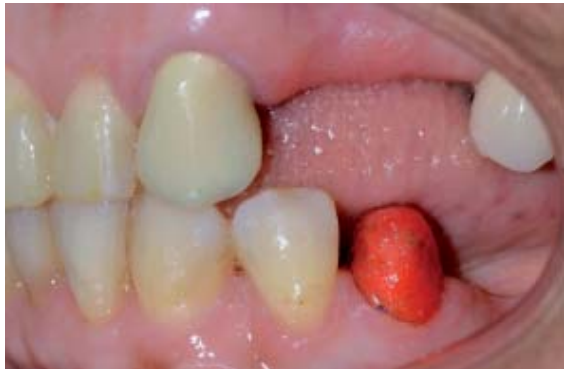

Figura 7. Cofia de acrílico

Luego se colocó acrílico Duralay sobre la cofia en las áreas que deban ser copiadas como el apoyo oclusal y placa proximal. Luego se asentó la PPR previamente aislada con vaselina, copiando la forma del apoyo y de la placa proximal (Figura 8). Se esperó la polimerización y se desgasto con una fresa para acrílico los excesos de Duralay dando forma a la cofia y verificando el correcto asentamiento a la base metálica de la PPR (Figura 9).

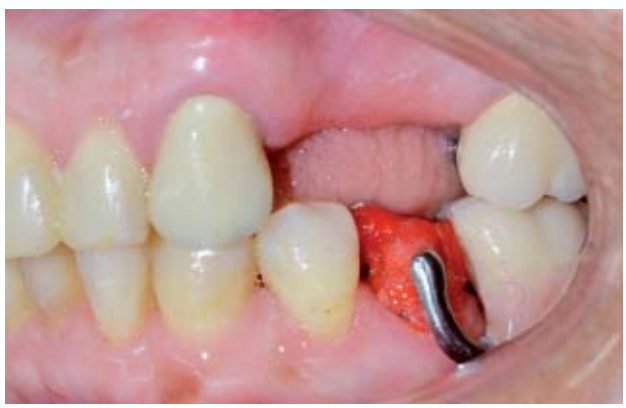

Figura 8. Adaptación en boca

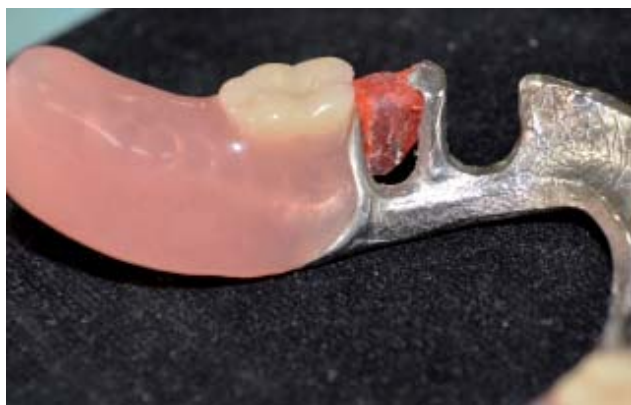

Figura 9. Verificando la adaptación

Se tomó una impresión a la pieza pilar con el provisional de acrílico cementada y se obtuvo un modelo (Figura 10) donde se realizó unas llaves de silicona por condensación pesada (Zetaplus, Zhermack) que le sirvió al laboratorio dental como referencia de contornos y altura (Figura 11 y 12).

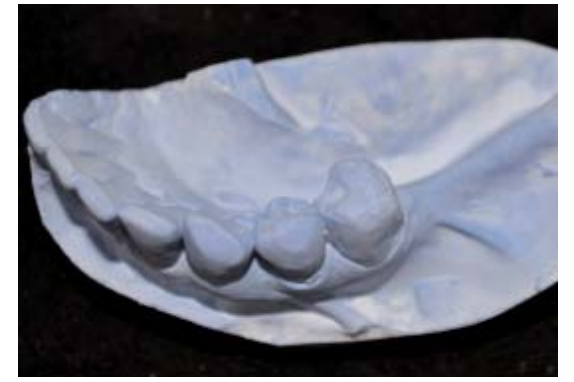

Figura 10. Modelo de corona provisional

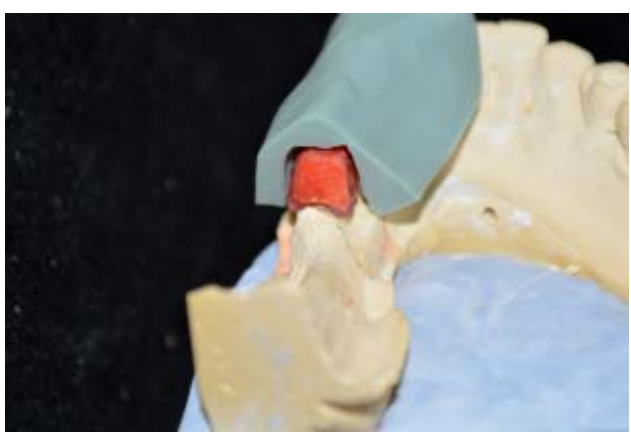

Figura 11. Llave de silicona para verificar contorno

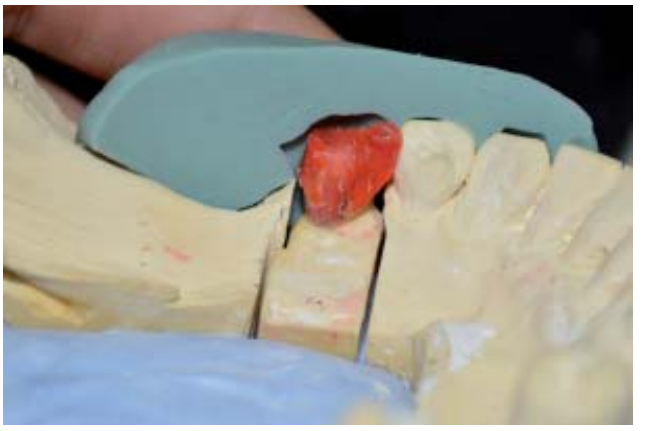

Figura 12. Llave de silicona para verificar altura

Se envió la cofia de acrílico con las llaves de silicona al laboratorio donde se realizó el procedimiento de colado en la aleación Níquel-Cromo verificando el espacio para la porcelana feldespática.

Luego se verificó la adaptación del colado de la cofia metálica en boca y el espacio para la posterior aplicación de la porcelana feldespática (Figura. 13).

Se escogió el color tomando como referencia las piezas adyacentes (Figura 14). 


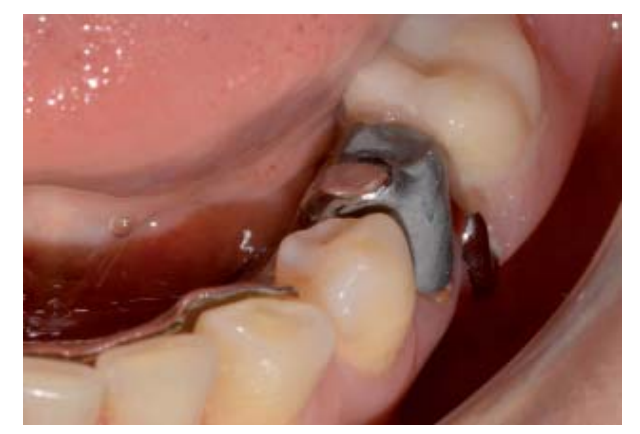

Figura 13. Verificación del colado.

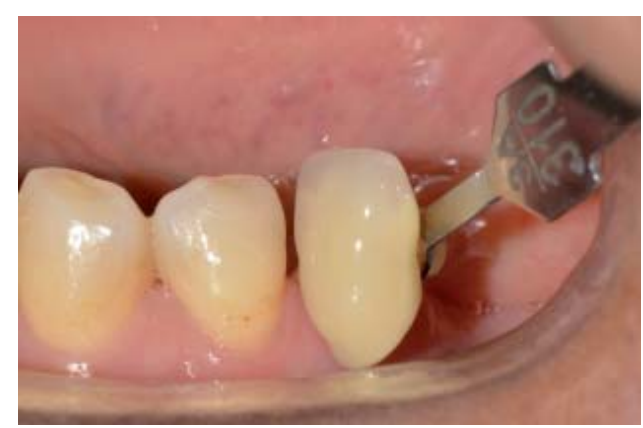

Figura 14. Selección de color

Una vez aplicada la porcelana se probó la corona en boca y nuevamente se verificó el asentamiento de la PPR y la retención del retenedor, el cual tuvo una calibración de 0.10 pulgadas. Se realizó ajustes de la porcelana para obtener una buena adaptación y retención (Figuras 15 y 16).

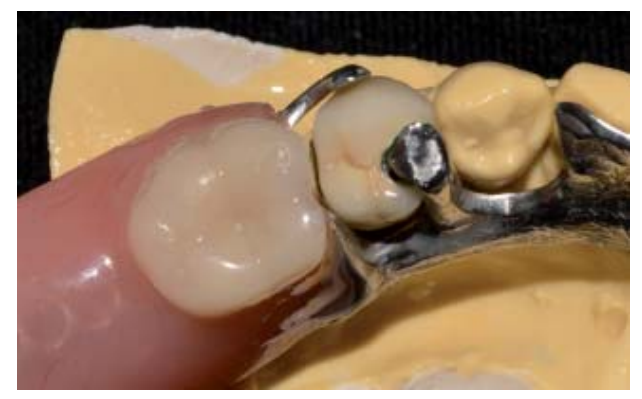

Figura 15. Adaptación de corona a PPR

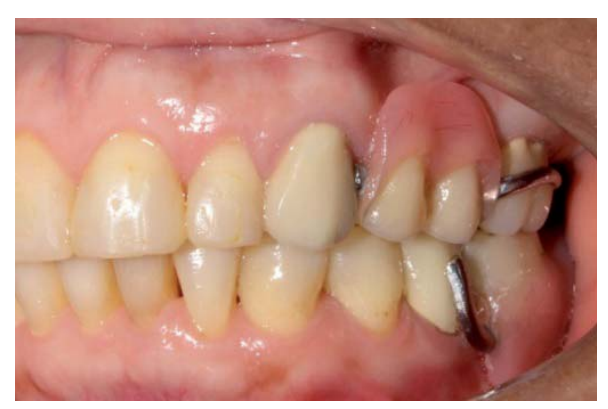

Figura16. Adaptación intraoral
Se realizó el glaseado de la cerámica feldespática, se cementó la corona metal cerámica y se instaló la PPR inferior (Figura 17).

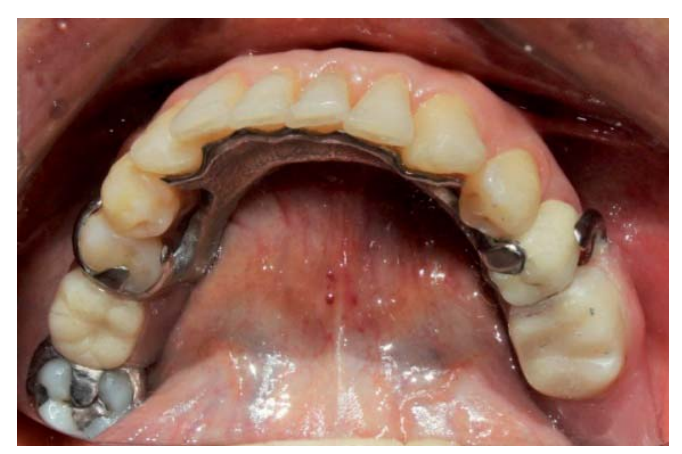

Figura 17. Cementación de corona

\section{DISCUSIÓN}

Diversas opciones de tratamiento para reemplazar dientes de pacientes parcialmente edéntulos están disponibles actualmente, estas incluyen prótesis removibles, prótesis fijas y prótesis implanto soportadas. Algunos pacientes que tienen la oportunidad de escoger entre prótesis implanto soportada y prótesis parcial removible, no se inclinan hacia los implantes por diversos motivos, otorgándole a la prótesis parcial removible una alta demanda. Esto también ocurre en casos donde las prótesis fijas no son indicadas en extensiones distales o en áreas edéntulas largas con gran pérdida ósea, la prótesis parcial removible puede ser planificada fácilmente como una opción de tratamiento (3).

El odontólogo es el responsable de decidir si la prótesis parcial removible se encuentra en buen o mal estado y si debe ser reemplazada o no. Si no necesita ser reemplazada se debe adaptar la nueva restauración a la PPR existente. Siendo esta una opción más económica y que requiere de menor tiempo (4).

Además de los métodos tradicionales de fabricación de las prótesis fijas, se requiere de solo una visita adicional del paciente al utilizar esta técnica, en la cual se observa la adaptación de la cofia de acrílico a los ganchos de la PPR (4).

Debido a que esta técnica no requiere el retiro de la PPR para el procedimiento de laboratorio, los pacientes no se ven incomodados (3), ya que por lo general 
este procedimiento involucra enviar la prótesis parcial removible al laboratorio dental para realizar los ajustes durante la inserción (5).

Con respecto a la confección de la corona, Burns y Unger nos indican que este es un procedimiento técnicamente demandante. La preparación de la pieza debe tener en cuenta la posición de todos los componentes y el eje de colocación de la prótesis parcial removible y al mismo tiempo seguir todos los parámetros asociados con la preparación dentaria propiamente dicha. Algunos factores a considerar incluyen la posición del diente en relación al plano oclusal propuesto con la prótesis parcial removible, el contorno vestibular y lingual deseado para los retenedores directos y el patrón de inserción y remoción propuesto para la prótesis parcial con respecto a la orientación del diente pilar así como de los dientes adyacentes (6).

El primer reporte de esta técnica se remonta a hace más de 35 años, donde Abdullah y Mulick reportan paso a paso la confección de una corona de oro con el objetivo de mantener la prótesis parcial removible en buen estado que presenta la paciente. Ellos también utilizan acrílico de combustión completa (DuraLay, CG) para la confección de una cofia que luego fue adaptada en boca junto con la prótesis parcial removible (7).

El uso de la resina acrílica (Duralay, CG) presenta diversas características y beneficios que la convierten en el material de elección para este tipo de procedimientos, entre ellos encontramos que presenta una baja contracción de polimerización otorgándole una gran precisión y alta estabilidad dimensional, copia fielmente los márgenes de las preparaciones, no presenta distorsión, su resistencia no se ve comprometida incluso en capas muy delgadas, fácil manipulación debido a sus propiedades tixotrópicas, alta humectabilidad del polvo y liquido fácilmente sin necesidad de mezclado, presenta un tiempo de trabajo de $4 \mathrm{mi}$ nutos y permite realizar correcciones indetectables y adiciones de material incluso después de su polimerización (8).

Helvey describe una técnica para reconstruir un pilar de PPR utilizando material polimérico (BelleGlass; Kerr) el cual tiene coeficiente de expansión térmica similar a la dentina y puede ser reparado con resina convencional si se produce desgaste de las superficies por el contacto con los ganchos (1).

Yoshinobu et al., describen una técnica de sustitución de la porción coronal de un pilar de prótesis parcial removible cuando la proporción corono raíz es desfavorable utilizando un atache magnético de $9 \mathrm{~N}$ de fuerza retentiva unido a un espigo intraradicular. Entre las ventajas descritas se encuentran la fuerza retentiva disponible, el espacio mínimo requerido y la eficiente disipación de fuerzas laterales hacia el pilar (9).

Marchack et al., promueven la confección de una cofia de zirconia utilizando la técnica CAD/CAM, digitalizando una cofia de resina acrílica confeccionada en boca sobre la imagen digital del pilar preparado. Las superficies de los ganchos, apoyos y placas proximales quedan en contacto con la zirconia y las superficies restantes son recubiertas por porcelana feldespática. La desventaja es la diferencia en el color de las cerámicas y la zirconia y el probable desgaste de la cofia de zirconia por la base metálica (10).

Otro reporte de caso publicado por Yoon y Chang indica que se puede restaurar dichos pilares también utilizando la técnica CAD/CAM pero digitalizando un modelo de diagnóstico con las preparaciones de los descansos y planos guías obtenido antes del deterioro de la pieza. Tae-Ho Yoon et al realizan una corona completa de disilicato de litio maquillada después del fresado, indicando que esta técnica es rápida y provee un alto resultado estético (5).

Livaditis en su investigación reporta la confección de análogos de los ganchos de la PPR para poder ser utilizados durante los procedimientos de laboratorio y la adaptación de la corona sea más exacta a todas las superficies metálicas. La técnica del análogo puede ser aplicada a todo tipo de coronas (metálicas completas o metal cerámicas) y todos los tipos de ganchos pueden ser replicados (11).

Los pasos detallados en este reporte de caso describen procedimientos sencillos que pueden ser realizados por el odontólogo general sin necesidad de contar con materiales o aparatos sofisticados. El beneficio principal de esta técnica es que el paciente puede conservar su prótesis removible durante todo el tiempo que tome la confección de la nueva corona, sin necesidad de ver perjudicada su eficacia masticatoria y estética por una posible ausencia de la prótesis. 
Con esta técnica sólo se requiere indicar al laboratorio dental que realice el envío de la cofia de acrílico antes de confeccionar el colado, con la finalidad de poder hacer la adaptación en boca de los espacios para el apoyo oclusal y la placa de contacto proximal que van a ir en contacto con la base metálica.

\section{CONCLUSIONES}

La técnica de adaptación de una corona metal cerámica a una prótesis parcial removible preexistente es una técnica simple de realizar y puede ser reproducida con éxito por cualquier odontólogo. Es importante determinar el estado de la prótesis parcial removible y de ser posible evitar su reemplazo para optimizar el tiempo y costo del tratamiento. También debemos evaluar el pronóstico del pilar y mantener la adaptación y sellado marginal de la corona, así como educar al paciente en la higiene y acudir a sus controles periódicos. La adaptación de las estructuras es fundamental para el pronóstico a largo plazo de esta técnica debido a que se evita la acumulación de placa y alimentos en las interfaces que puede llevar al fracaso del tratamiento.

\section{Correspondencia}

\section{Melissa Torres Herbozo}

Correo electrónico: tmelissa4@hotmail.com

\section{REFERENCIAS BIBLIOGRAFICAS}

1. Helvey GA. Retrofitting crowns to an existing removable partial denture clasp: A simple technique. J Prosthet Dent. 2002; 87(4):399-402.

2. Lee $\mathrm{H}$, Shirakura A. A technique to fabricate a cast metal crown for an existing removable partial denture using an acrylic resin template. J Prosthet Dent. 2007;97(3):181-2.

3. Saluja BS, Mittal D. An overview of removable partial denture. Indian J Dent SCI. 2012;3(4):117-23.

4. Fujisawa M, Adachi K, Tsuruta S, Ishibashi L. Procedure for fitting a fixed partial denture to an existing removable partial denture. J Prosthet Dent. 2004:91(4):392-4.

5. Yoon TH, Chang WG. The fabrication of a CAD/ CAM ceramic Crown to fit an existing partial removable dental prosthesis: A clinical report. J Prosthet Dent. 2012;108(3):143-6.

6. Burns DR, Unger JW. The construction of crowns for removable partial denture abutment teeth. Quintessen- ce Int. 1994;25(7):471-5.

7. Abdullah SI. Mulick SC. A new Crown for an existing removable partial prosthetics. Quintessence Int. 1979;4(10):35-40.

8. Gibbs SB, Versluis A, Tantbirojn D, Ahuja S. Comparison of polymerization shrinkage of pattern resins. $\mathrm{J}$ Prosthet Dent. 2014;112(2):293-8.

9. Maeda Y, Nakao K, Yagi K, Matsuda S. Composite resin root coping with a keeper for magnetic attachment for replacing the missing coronal portion of a removable partial denture abutment. J Prosthet Dent. 2006;96(2):139-42.

10. Marchack BM, Chen LB, Marchack CB, Futatsuki Y. Fabrication of an all-ceramic abutment crown under an existing removable partial denture using CAD/ CAM technology. J Prosthet Dent. 2007;98(6):47882.

11. Livaditis G. Fabricating abutment crowns for existing removable partial dentures using custom resin clasp analogs. J Prosthet Dent. 1998;80(5):619-29.

Recibido : 31/03/2015

Aceptado: 15/07/2015 